\title{
COSMOLOGICAL EFFECTS OF WEIBEL-TYPE INSTABILITIES
}

\author{
M. LAZAR ${ }^{1}$, R. SChLicKeISER ${ }^{2}$, R. WIELEBINSKi ${ }^{3}$, AND S. POEDTS ${ }^{1}$ \\ ${ }^{1}$ Center for Plasma Astrophysics, Celestijnenlaan 200B, 3001 Leuven, Belgium; Marian.Lazar@wis.kuleuven.be \\ ${ }^{2}$ Institut für Theoretische Physik, Lehrstuhl IV: Weltraum- und Astrophysik, Ruhr-Universität Bochum, D-44780 Bochum, Germany \\ ${ }^{3}$ Max-Planck-Institut für Radioastronomie, D-53121 Bonn, Germany \\ Received 2008 September 30; accepted 2008 December 1; published 2009 March 5
}

\begin{abstract}
New arguments are given here in favor of Weibel-type instabilities as one of the most plausible sources of the cosmological magnetic field. The Weibel instability has recently been proposed as one of the secondary mechanisms of relaxation for the large interpenetrating formations of galactic and intergalactic plasma. Here, these investigations are extended to counterstreaming plasmas which have, in addition, intrinsic temperature anisotropies, and where any form of the Weibel-type instability can be excited. This can be a simple filamentation instability due to the relative motion of counterstreaming plasmas, or a Weibel-like instability when it is generated by an excess of transverse temperature with respect to the streaming direction. But it can also be a cumulative filamentation/Weibel instability when the plasma is hotter along the streaming direction. Such plasma systems are relevant for the relative motions of filaments and sheets of galaxies, and are expected to exist at large scales and any age of our Universe. For such counterstreaming plasmas with internal temperature anisotropies, any Weibel-type instability mentioned before can become the primary wave relaxation mechanism of the plasma anisotropy, because it develops easily faster than the principal competitor, which is the two-stream electrostatic instability. The estimations made here for typical parameters of intergalactic plasmas, provide micro-Gauss levels of the magnetic field of Weibel type, which are consistent with magnetic field values, $10^{-7}-10^{-5} \mathrm{G}$, derived from Faraday rotation measure of the linearly polarized emission of galactic or extragalactic sources.
\end{abstract}

Key words: cosmology: theory - large-scale structure of universe - magnetic fields - plasmas

\section{INTRODUCTION}

Magnetic fields are widely present throughout the universe, with strengths ranging from 0.1 to a few micro-Gauss $(\mu \mathrm{G})$ in galactic clusters, to less than $1 \mathrm{G}$ near the Earth, or up to $10^{14} \mathrm{G}$ in the magnetosphere of neutron stars. Galactic and extragalactic magnetic fields have been extensively investigated and a large amount of observational data has been reported in the literature. The interested readers may find details in some review works, e.g., Kronberg (1994), Widrow (2002), and Wielebinski \& Beck (2005).

At present, the origin of the cosmic magnetic field is unknown. The dynamo principle was first invoked by Parker (1955) and then by Steenbeck \& Krause (1968) and Vainshtein \& Ruzmaiken (1971) to explain the origin of galactic magnetic fields. According to this principle, the energy associated with the differential rotation of spiral galaxies is converted into magnetic field energy. Indeed, dynamos are able to regenerate largescale magnetic fields continuously. However, in the dynamo processes the induced currents amplify and maintain the initial magnetic field seed that must be already present for this action. Since very weak cosmological fields can act as seeds for the galactic dynamo, the creation mechanism of even the tiniest cosmological field would help complete the dynamo paradigm.

The cosmic string network was invoked as one plausible mechanism for the magneto-genesis. It is based on the generation of vorticity in the primordial plasma, which is subsequently converted to a magnetic field through the Harrison-Rees mechanism (Harrison 1970; Rees 1987). However, this scenario is conditioned by the hypothetical existence of cosmic strings with enough tension (not discovered yet), and such string-driven seed fields would explain $\mu \mathrm{G}$ magnetic fields observed today only after an efficient galactic dynamo for amplification (Battefeld et al. 2008).
Intergalactic space is filled with a hot dilute plasma $(T \simeq$ $10^{7}-10^{8} \mathrm{~K}, n \simeq 10^{-4} \mathrm{~cm}^{-3}$ ), and its existence has important consequences on the formation and evolution of the galaxies and the universe. Currently, the best knowledge of the properties of intergalactic matter comes from studies of the diffuse radio and X-ray emission from clusters of galaxies. Clusters of galaxies act as gravitational potential wells in which intergalactic matter clumps. By combining observations of the diffuse radio (Schlickeiser et al. 1987) and hard X-ray emission from the Coma cluster of galaxies (Taylor et al. 1994), the value of the average magnetic field strength in intergalactic space has been found to be between 0.04 and $12 \mu \mathrm{G}$.

Theoretically, electromagnetic instabilities of the Weibel type are capable to create the seed magnetic field in the large interpenetrating structures of intergalactic plasmas (IGP), even when they are competed by the electrostatic two-stream instability (TSI) (Schlickeiser \& Shukla 2003; Okabe \& Hattori 2003). Moreover, numerical simulations (Sakai et al. 2004; Fujita \& Kato 2006; Medvedev et al. 2006) have confirmed that the Weibel-type instabilities can create quasi-static and strongly structured magnetic field in colliding cosmological plasmas (like shocks, etc). Moreover, in order to explain the observed intergalactic magnetic field, the turbulence excited during the processes of large-scale structure formation can amplify seed fields as strong as $B \geqslant 10^{-10} \mathrm{G}$ (Brügen et al. 2005).

Plasma instabilities arise and release the excess of free energy stored in the plasma particle anisotropy, residing, e.g., in a temperature anisotropy or in a counterstreaming motion of the plasma. A substantial fraction of the kinetic energy of the plasma particles is converted by the electromagnetic instabilities contributing to the generation and amplification of magnetic energy (Califano et al. 1998). Thus, the spontaneous magnetization of a plasma with a temperature anisotropy is attributed to the Weibel instability (Weibel 1959), whereas the 
magnetization of a counterstreaming plasma or a beam-plasma system is associated with the filamentation instability (FI; Fried 1959). Schlickeiser \& Shukla (2003) have used a beam-plasma model, with a cold electron beam propagating through a hot electron-ion plasma with isotropic Maxwellian distributions. Such a plasma system is unstable against the excitation of two characteristic instabilities: the FI, which is electromagnetic and propagates perpendicular to the streams, and the two-stream (electrostatic) instability, which propagates along the streaming direction and is, in general, much faster than the FI. Indeed, for typical conditions of cosmological structure formation (Miniati 2002), the TSI develops first and relaxes the beam-plasma system to an anisotropic bi-Maxwellian plasma (Schlickeiser $\&$ Shukla 2003). But such a temperature anisotropy will relax by the excitation of the Weibel instability which propagates perpendicular to the direction of the highest temperature (Weibel 1959). In this case, the magnetic field will reach saturation maximum values as close to $3.4 \times 10^{-7} \mathrm{G}$, which can explain the existence of the localized intergalactic magnetic fields of $\mu \mathrm{G}$ strength.

More recently, it has been shown that the FI becomes faster and the growth rate of the magnetic field can be enhanced by the intrinsic temperature anisotropy of counterstreaming plasmas. This is exactly the case of a hotter plasma along the streaming direction (Lazar 2008), for which the contributions of the FI and the Weibel-like instability (WI) cumulate and lead to an enhancing effect of the magnetic instability (Lazar et al. 2006; Stockem \& Lazar 2008). Otherwise, if the plasma kinetic energy perpendicular to the streams exceeds the parallel kinetic energy, the anisotropy in the velocity space decreases and becomes less effective, so that the FI is reduced or even suppressed (Bret \& Deutsch 2006; Lazar et al. 2006; Stockem \& Lazar 2008). However, in this case a Weibel-like mode is expected to arise along the streaming direction (Bret et al. 2004), and this could be more efficient than the FI in the process of magnetization. These instabilities of Weibel type are the fastest and the most efficient relaxation mechanism for the initial plasma anisotropy, as long they saturate at maximum growth rates comparable to or larger than those of other plasma instabilities, e.g., the TSI. These new scenarios approve and further sustain the role of Weibel instabilities as one of the main sources of magnetic energy in astrophysical plasmas. For relativistic streams, it has been also shown that the fastest growing mode is a mixture of longitudinal and transverse fields with an intermediate oblique orientation of the wavevector (Bret et al. 2005). However, in the case of slow nonrelativistic plasma streams this branch aligns to streaming axis and its growth rate decreases and fits to that of the TSI.

There is observational evidence for the fact that the IGP is sufficiently warm $\left(T \simeq 10^{7} \mathrm{~K}\right)$, and in the most successful scenario of the cosmological structure formation, the large-scale structures, like filaments and sheets of galaxies, evolve by the gravitational collapse of initially over-dense regions giving rise to an intense relative motion of fully ionized gaseous matter with a maximum speed of the order $v_{0} \simeq 10^{4} \mathrm{~km} \mathrm{~s}^{-1}$ (see, e.g., Schlickeiser \& Shukla (2003), and references therein). Thus, the IGP is supposed to be dominated by a variety of velocity anisotropies, provided internally either by an anisotropic kinetic energy or by the externally imposed drifts. Moreover, the magnetic field generated by the Weibel mechanism can evolve at large space scales (Schlickeiser 2005), and they could therefore offer a plausible explanation for the existence of the highly variable large-scale structures with reversals of direction of the galactic and intergalactic magnetic fields, as was shown

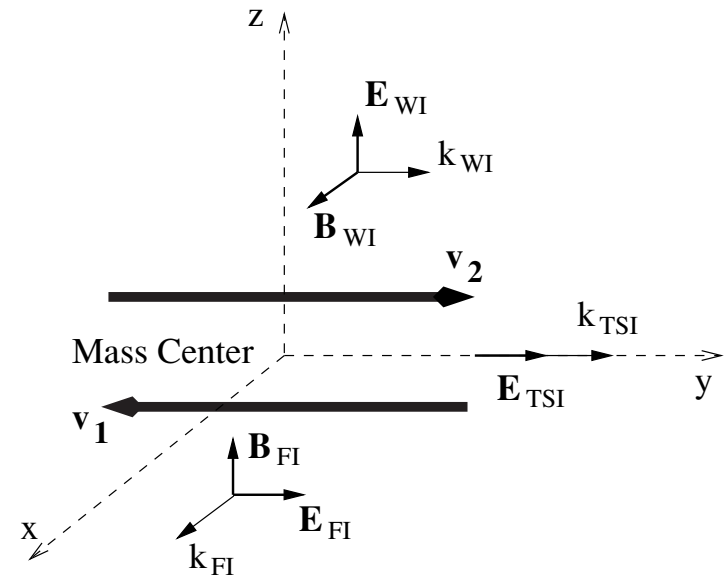

Figure 1. Two counterstreaming plasmas along the $y$-axis, and the unstable modes developing in the system: the electromagnetic Weibel-like mode (labeled with "WI") and "TSI" both of them propagating along the streaming direction, and the "FI" propagating perpendicular to the streaming direction.

recently in high-resolution observations (see the maps of Han et al. 2006, and references therein).

In the present paper, we extend the investigation of the magnetic instabilities of Weibel type and we study how efficient these instabilities are in the process of the magnetization of interpenetrating plasmas with intrinsic temperature anisotropies and with properties that are relevant for the complex behavior of IGP and the cosmological structure formation (Schlickeiser \& Shukla 2003). We evaluate this efficiency by determining first their growth rates, and for conditions under which they are competitive with respect to the TSI, we calculate the maximum values of the Weibel-type magnetic fields reached at the saturation. Looking to the parameters of the IGP mentioned before, we should remark that the plasma temperature and the typical streaming velocities are sufficiently low to neglect relativistic effects. Moreover, for nonrelativistic plasma temperatures there is no noticeable difference between the covariant and noncovariant growth rates (Schlickeiser 2004; Schaefer-Rolffs \& Schlickeiser 2005).

\section{DISPERSION THEORY}

In Figure 1, we fix the orientation for the electromagnetic instabilities (filamentation and Weibel) and the electrostatic (two-stream) unstable modes, which arise in a counterstreaming plasma with internal temperature anisotropies. Such a plasma system is described here by the distribution function

$$
f_{0}\left(v_{x}, v_{y}, v_{z}\right)=\frac{1}{2 \pi^{3 / 2} v_{\mathrm{th}}^{2} v_{\mathrm{th} \perp}} e^{-\frac{v_{x}^{2}+v_{z}^{2}}{v_{\mathrm{th}}^{2}}}\left[e^{-\frac{\left(v_{y}+v_{0}\right)^{2}}{v_{\mathrm{th} \perp}^{2}}}+e^{-\frac{\left(v_{y}-v_{0}\right)^{2}}{v_{\mathrm{th} \perp}^{2}}}\right] .
$$

For the sake of simplicity, we assume that the two interpenetrating plasmas are symmetric and have the same properties (a condition frequently satisfied with respect to the mass center): equal densities, $\omega_{p, e, 1}=\omega_{p, e, 2}=\omega_{p, e}$, equal but opposite streaming velocities, $\left|v_{1}\right|=v_{2}=v_{0}$, and the same temperature parameters, i.e., thermal velocities, $v_{\text {th }, x, 1}=v_{\text {th }, z, 1}=$ $v_{\text {th }, x, 2}=v_{\text {th }, z, 2}=v_{\text {th }}, v_{\text {th }, y, 1}=v_{\text {th }, y, 2}=v_{\text {th }, y}$, and thermal anisotropies, $A_{1}=A_{2}=A=\left(v_{\text {th }, y} / v_{\text {th }}\right)^{2}-1$. Considering symmetric counterstreaming plasmas, with the same densities, velocities, and temperature parameters, enables us to work with 
simple forms of the dispersion relations and only with the reactive (purely growing) part of their unstable solutions $(\operatorname{Re} \omega=0$ and $\operatorname{Im} \omega=\Gamma>0$ ). This is what we need, a minimal resonant (Landau) dissipation on the plasma particles, in order to find the maximum potential of growth of the TSI, and to compare to that of the FI and the WI, which are, in general, nonresonant.

The counterstreaming plasma system is unstable against the excitation of the electrostatic TSI and the electromagnetic WI. The general dispersion relation derived from the VlasovMaxwell equations takes the form (Bret et al. 2004; Fiore et al. 2006)

$$
\operatorname{det}\left|\frac{\omega^{2}}{c^{2}} \epsilon_{i j}+k_{i} k_{j}-k^{2} \delta_{i j}\right|=0,
$$

where the components of the permittivity tensor are given by

$\epsilon_{i j}=\delta_{i j}+\sum_{a} \frac{\omega_{p, a}^{2}}{\omega^{2}}\left[\int d v^{3} v_{i} \frac{\partial f_{0, a}}{\partial v_{j}}+\int d v^{3} v_{i} v_{j} \frac{\mathbf{k} \cdot \partial f_{0, a} / \partial \mathbf{v}}{\omega-\mathbf{k} \cdot \mathbf{v}}\right]$.

The unperturbed velocity distribution function $f_{0, a}(\mathbf{v})$ (for the particles of species $a$ ) is normalized by $\int d \mathbf{v} f_{0, a}(\mathbf{v})=1$, and $\omega_{p, a}=\left(4 \pi n_{a} e^{2} / m_{a}\right)^{1 / 2}$ is the plasma frequency.

Next, we describe the unstable solutions of Equation (2), which are responsible for the relaxation of the counterstreaming plasmas with temperature anisotropies.

\subsection{Unstable Modes with $\boldsymbol{k} \| \boldsymbol{v}_{0}$}

\subsubsection{Weibel-like Instability $\left(\boldsymbol{k} \cdot \boldsymbol{E}=0, v_{t h}>v_{t h, y}\right)$}

For a plasma system with a finite temperature, there is an important departure from the cold plasma model where no transverse modes could interact with the electrons for wavevectors parallel to the streaming direction, $\mathbf{k} \| \mathbf{v}_{\mathbf{0}}$, as no electrons move perpendicularly to the streams. Such electrons are introduced by a nonvanishing transverse temperature of the plasma counterstreams and the electromagnetic modes propagating along the streaming direction can be excited (Bret et al. 2004).

Thus, considering first the unstable modes with a wavevector parallel to the streaming direction, $k=k_{y}$, and using the distribution function (1), the dispersion relation (2) simplifies to

$$
\left(\frac{\omega^{2}}{c^{2}} \epsilon_{x x}-k_{y}^{2}\right)\left(\frac{\omega^{2}}{c^{2}} \epsilon_{z z}-k_{y}^{2}\right) \epsilon_{y y}=0,
$$

which admits three solutions. Due to the symmetry of the system the first two branches of the transverse modes are described by the dispersion relation

$$
\begin{aligned}
\frac{k_{y}^{2} c^{2}}{\omega^{2}} & =\epsilon_{x x}^{\mathrm{WI}}=\epsilon_{z z}^{\mathrm{WI}} \\
& =1-\frac{\omega_{p, e}^{2}}{\omega^{2}}\left\{1-\frac{1}{A+1}\left[1+\frac{1}{2}\left(f_{1} Z\left(f_{1}\right)+f_{2} Z\left(f_{2}\right)\right)\right]\right\},
\end{aligned}
$$

which is written here in terms of the well-known plasma dispersion function (Fried \& Conte 1961)

$$
Z(f)=\pi^{-1 / 2} \int_{-\infty}^{\infty} d x \frac{\exp \left(-x^{2}\right)}{x-f}, \quad \text { with } \quad f_{1,2}=\frac{\omega \mp k_{y} v_{0}}{k_{y} v_{\mathrm{th}, y}}
$$

Equation (5) describes the electromagnetic Weibel mode as was shown in some temperature-dependent investigations of beamplasma systems (Okada et al. 1977; Bret et al. 2004). The existence of the Weibel instability is limited to wavenumbers less than the cutoff value

$$
k_{y, c}^{\mathrm{WI}}=\frac{\omega_{p, e}}{c}\left\{\frac{1}{A+1}\left[1+\frac{v_{0}}{v_{\mathrm{th}, y}} \operatorname{Re} Z\left(\frac{v_{0}}{v_{\mathrm{th}, y}}\right)\right]-1\right\}^{1 / 2},
$$

which must be a real (not complex) solution of Equation (5) in the limit of $\Gamma(k)=\operatorname{Im} \omega(k)=0$. We should observe that for $v_{0} \gg v_{\text {th, } y}$, i.e., for sufficiently cold plasma streams, the cutoff wavenumber becomes imaginary and the instability quenches. In cold or thermally isotropic plasmas the electromagnetic mode is stable, but it can be destabilized by temperature effects (Weibel like), especially due to a nonvanishing temperature transverse to the streams, $v_{\text {th }}>v_{\text {th }, y}$.

$$
\text { 2.1.2. } \operatorname{TSI}(\boldsymbol{k} \times \boldsymbol{E}=0)
$$

The TSI is an electrostatic unstable mode propagating along the streaming direction, and described by

$$
\epsilon_{y y}^{\mathrm{TSI}}=1+\frac{\omega_{p, e}^{2}}{k_{y}^{2} v_{\mathrm{th}, y}^{2}}\left[2+f_{1} Z\left(f_{1}\right)+f_{2} Z\left(f_{2}\right)\right]=0 .
$$

The TSI is sensitive only to the parallel thermal velocities and their spread reduces the growth rate of the instability. Thus, the TSI a maximal efficiency in the process of relaxation only for low temperature plasmas, $v_{\text {th, } y} \ll v_{0}$. Under this condition, the arguments $f_{1,2}>1$ are sufficiently large, and we can use the asymptotic approximation for the plasma dispersion function in Equation (8) and find the cutoff wavenumber of the TSI,

$$
k_{y, c}^{\mathrm{TSI}}=\frac{\omega_{p, e}}{v_{0}}\left(1+\frac{3}{2} \frac{v_{\mathrm{th}, y}^{2}}{v_{0}^{2}}\right)^{1 / 2} \simeq \frac{\omega_{p, e}}{v_{0}}\left(1+\frac{3}{4} \frac{v_{\mathrm{th}, y}^{2}}{v_{0}^{2}}\right) .
$$

For a negligible thermal spread, $v_{0} \gg v_{\text {th, } y}$ (cold plasmas), the cutoff wavenumber will depend only on the streaming velocity $k_{y, c}^{\mathrm{TSI}} \rightarrow \omega_{p, e} / v_{0}$.

\section{2. $F I\left(k \perp v_{0}\right)$}

We now consider the electromagnetic modes propagating perpendicular to the streaming direction, $k=k_{x}$. The dispersion relation (2) then becomes

$$
\epsilon_{x x}\left(\frac{\omega^{2}}{c^{2}} \epsilon_{y y}-k_{x}^{2}\right)\left(\frac{\omega^{2}}{c^{2}} \epsilon_{z z}-k_{x}^{2}\right)=0,
$$

which has also three branches of solutions, but only one describes the filamentation unstable mode, namely

$$
\begin{aligned}
& \frac{k_{x}^{2} c^{2}}{\omega^{2}}=\epsilon_{y y}^{\mathrm{FI}} \\
& =1-\frac{\omega_{p, e}^{2}}{\omega^{2}}\left\{1-\left(A+1+2 \frac{v_{0}^{2}}{v_{\mathrm{th}}^{2}}\right)\left[1+\frac{\omega}{k_{x} v_{\mathrm{th}}} Z\left(\frac{\omega}{k_{x} v_{\mathrm{th}}}\right)\right]\right\} .
\end{aligned}
$$

The FI is a solution of Equation (11), and it is restricted to wavenumbers less than a cutoff value given by

$$
k_{x, c}^{\mathrm{FI}}=\frac{\omega_{p e}}{c}\left[A+2\left(\frac{\beta_{0}}{\beta_{\mathrm{th}}}\right)^{2}\right]^{1 / 2} .
$$


In this case, the Weibel effect due to the temperature anisotropy cumulates to the FI, and yields either an enhancing of the growth rate or a quenching of the magnetic instability, depending on the plasma kinetic energy along the streaming direction, and, in particular, whether this exceeds the perpendicular kinetic energy or not (Lazar et al. 2006; Stockem \& Lazar 2008).

\section{NUMERICAL SOLUTIONS}

The most efficient wave mode capable to release the excess of free energy and to relax the counterstreaming distribution (1), will be the fastest wave mode, i.e., the mode with the highest maximum growth rate. We, therefore, proceed first with a numerical evaluation of the growth rates for the unstable modes described before. For the streaming velocity we use the typical maximal value estimated from the observations as $v_{0}=10^{7} \mathrm{~m}$ $\mathrm{s}^{-1}$ (Miniati 2002; Schlickeiser \& Shukla 2003). There are two situations of interest depending on the feature of the temperature anisotropy. For higher transverse temperatures (with respect to the streaming direction) we expect an important contribution from the Weibel-like mode propagating parallel to the streams. Otherwise, when the plasma is hotter along the streaming direction, the anisotropy increases and we expect an enhancing cumulative effect of the filamentation and Weibel instabilities propagating in this case perpendicular to the streams. Both of these two cases must be relevant in a cosmological scenario with colliding plasma shells which exhibit internal temperature anisotropies.

\subsection{TSI}

The growth rates of the two-stream electrostatic instability are solutions of Equation (8) and they are plotted in Figure 2 for different thermal velocities along the streaming direction, namely $v_{\text {th }, y} / v_{0}=0.0 ; 0.1 ; 0.5 ; 1.0 ; 10.0$. For low thermal velocities, $v_{\text {th }, y}=0.1 v_{0} \ll v_{0}=10^{7} z \mathrm{~m} \mathrm{~s}^{-1}$, the instability is still strong so that the growth rates are comparable to those obtained for cold counterstreaming plasmas (see the dotted line in Figure 2). We note that the thermal velocity spread of plasma particles is increased by the instability itself (Melrose 1989), but the effect of a higher thermal spread will limit or even suppress the instability. In Figure 2, we remark again that for a sufficiently large thermal velocity, for example, $v_{\mathrm{th}, y}=10 v_{0}$, the reactive instability disappears and damping occurs instead.

\subsection{Counterstreaming Plasmas with $v_{\text {th }}>v_{\text {th, } y}$}

We now consider the Weibel-like mode described by Equation (5). The growth rates, $\operatorname{Im} \omega(k)=\Gamma(k)>0$, are plotted with dashed lines in Figure 3 in terms of the wavenumber $k$, and for diverse temperature anisotropies. The Weibellike mode arises only for sufficiently large transverse temperatures, $v_{\text {th }}>v_{\text {th }, y}$. In this case, the temperature anisotropy will take negative and subunitary values $-1<A=T_{y} / T-1=$ $\left(v_{\text {th }, y} / v_{\text {th }}\right)^{2}-1<0$.

Because of the streaming motion of the plasma, the Weibellike mode looks in this case different than the classical instability of Weibel (1959). In Figure 3(b), for higher plasma temperatures, the growth rates exhibit slope breaks for wavenumbers larger than a threshold value that depends on the temperature anisotropy, and where the purely growing instability changes and becomes a fully propagating mode that propagates not only in space but in time as well, $\omega_{r} \neq 0$. This is a new regime of the Weibel-like mode that seems to be typically encountered

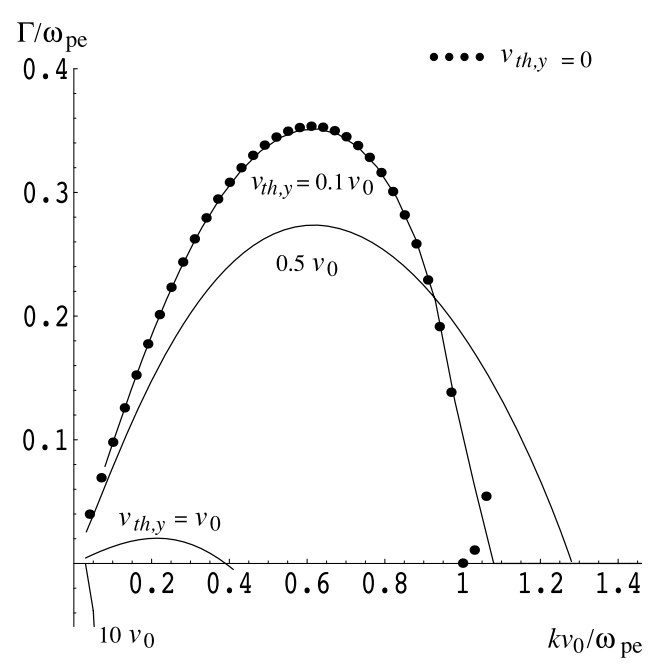

Figure 2. Numerical evaluation for the growth rates of the two-stream electrostatic instability $\left(v_{0} \simeq 10^{7} \mathrm{~m} \mathrm{~s}^{-1}\right)$, which are solutions of Equation $(8)$. For sufficiently low temperatures $\left(v_{\text {th }, y} \leqslant 0.1 v_{0}\right)$ the growth rates practically coincide with those obtained for cold counterstreaming plasmas (dotted line).

in a counterstreaming plasma system with intrinsic temperature anisotropies. In our study here, only the maximum growth rates reached at the saturation are important, and therefore, these aspects remain to be investigated in detail elsewhere.

Here our analysis splits in two cases. In Figure 3(a), the temperature perpendicular to the streams is assumed to be constant and close to the temperature of the IGP, $v_{\text {th }} \simeq 10^{7} \mathrm{~m}$ $\mathrm{s}^{-1}$. The WI arises for a smaller thermal velocity along the streaming direction, and the growth rates are plotted for three values of $v_{\text {th }, y} / v_{\text {th }}$, ranging from 0.1 to 0.4 .

On the other hand, in Figure 3(b) we keep the temperature constant along the streaming direction and assume that it approaches the temperature of the IGP, $v_{\text {th, } y} \simeq 10^{7} \mathrm{~m} \mathrm{~s}^{-1}$, and plot the growth rates of the WI for higher transverse temperatures. In this case, the instability becomes significantly faster. Taking a large but reasonable temperature anisotropy, for example $A=-0.99$, which corresponds to a thermal velocity $v_{\text {th }}=10^{8} \mathrm{~m} \mathrm{~s}^{-1} \simeq 0.33 c$ (where $c=3 \times 10^{8} \mathrm{~m} \mathrm{~s}^{-1}$ is the speed of light in vacuum), and a relativistic Lorentz factor $\gamma_{\mathrm{th}}=\left(1-v_{\mathrm{th}}^{2} / c^{2}\right)^{-1 / 2} \simeq 1.1$, the growth rate will reach an important maximum value $\Gamma \simeq 0.17 \omega_{p e}$.

The fully nonrelativistic plasma temperatures are usually limited to less than $5 \mathrm{keV}$ (Bergman \& Eliasson 2001), which means thermal velocities $v_{\text {th }} \leqslant 0.15 c$. However, quantitatively, the relativistic effects become important only for a kinetic energy of the plasma electrons comparable or larger than their energy at rest, $m_{0} c^{2} \simeq 500 \mathrm{keV}$, which corresponds to a thermal velocity $v_{\text {th }} \simeq 2 \times 10^{8} \mathrm{~m} \mathrm{~s}^{-1}$ and a Lorentz factor $\gamma_{\text {th }} \simeq 1.34$. Therefore, for a Lorentz factor still close to unity we can extend and use the approach developed here, to estimate how fast this instability can be in the limit of weakly-relativistic temperatures, $v_{\text {th }}<2 \times 10^{8} \mathrm{~m} \mathrm{~s}^{-1}$.

Moreover, the case chosen in Figure 3(b) seems to be more realistic for the counterstreaming structures of the IGP because the streams can be naturally created at temperatures not far from the IGP temperature, $T \simeq 10^{7} \mathrm{~K}$, and their transverse temperature is increased by the self-generated magnetic field, which pinches the beam radially and prevents the simple outward movement of beam particles. For a lower temperature anisotropy, $v_{\mathrm{th}, y} \rightarrow v_{\mathrm{th}}$, see, for example, in Figure 3(a), the instability diminishes $\left(\Gamma \ll \omega_{p, e}\right)$, and instead damping occurs $(\Gamma<0)$. 

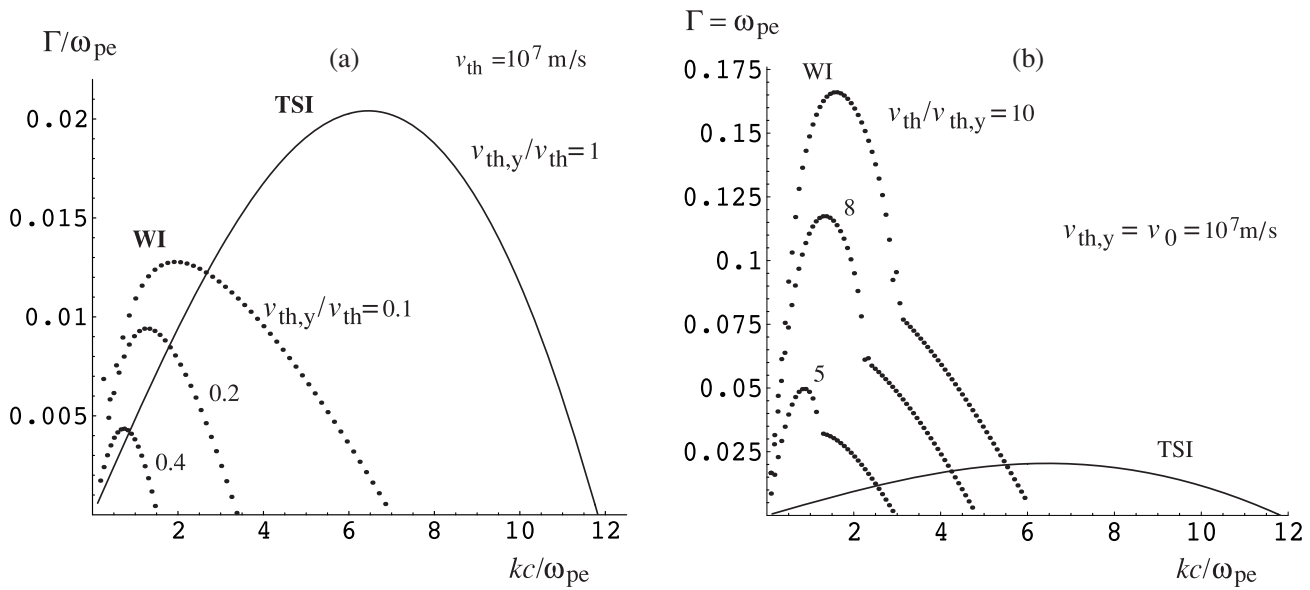

Figure 3. Numerical evaluation of the growth rates of Weibel instability described by Equation (5). In $(a)$ the transverse temperature with respect to the streaming direction approaches the average temperature of IGP, so that $v_{\text {th }} \simeq 10^{7} \mathrm{~m} \mathrm{~s}^{-1}$, and the temperature along the streaming direction is smaller: $v_{\text {th }, y} / v_{\text {th }}=0.1 ; 0.2 ; 0.4$. In $(b)$ we change and assume $v_{\text {th }, y} \simeq 10^{7} \mathrm{~m} \mathrm{~s}^{-1}$, and $v_{\text {th }}$ much larger as given by $v_{\text {th }} / v_{\text {th }, y}=5 ; 8 ; 10$.
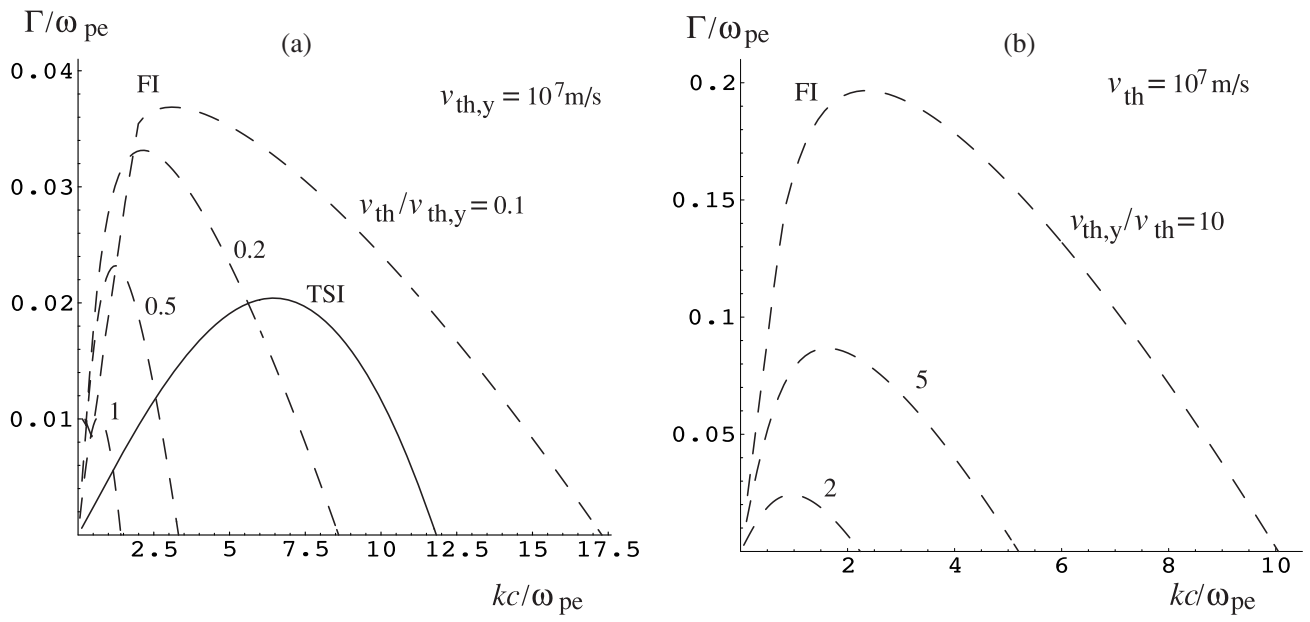

Figure 4. Numerical evaluation of the growth rates for the filamentation unstable mode described by Equation (11). In (a) the parallel temperature with respect to the streaming direction is considered close to the average temperature of IGP, so that $v_{\mathrm{th}, y} \simeq 10^{7} \mathrm{~m} \mathrm{~s}^{-1}$, but it is larger than the transverse temperature, so that $v_{\text {th }} / v_{\text {th, } y}=$ $0.1 ; 0.2 ; 0.5 ; 1$. In $(b)$ we change and assume $v_{\text {th }} \simeq 10^{7} \mathrm{~m} \mathrm{~s}^{-1}$, and less than $v_{\text {th }, y}$, given by $v_{\text {th }, y} / v_{\text {th }}=2 ; 5 ; 10$.

\subsection{Counterstreaming Plasmas with $v_{t h, y}>v_{t h}$}

The growth rates of the FI are shown in Figure 4. They are the purely growing solutions of Equation (11), $\operatorname{Im} \omega(k)=\Gamma(k)>0$ (and $\operatorname{Re} \omega=0$ ), which cumulate the Weibel effect produced by the temperature anisotropy, $A \neq 0$.

The two cases considered here are similar to those from Figure 3. In Figure 4(a) the temperature parallel to the streaming direction is assumed to be close to the temperature measured in the IGP, $v_{\text {th }, y} \simeq 10^{7} \mathrm{~m} \mathrm{~s}^{-1}$, and we calculate numerically the growth rates for smaller values of the transverse temperature, $v_{\text {th }} / v_{\text {th }, y}=0.1 ; 0.2 ; 0.5 ; 1.0$. In Figure $4(\mathrm{~b})$ we keep the transverse temperature constant at $v_{\text {th }}=10^{7} \mathrm{~m} \mathrm{~s}^{-1}$, and the growth rates are plotted for larger values of the parallel thermal velocity $v_{\text {th, } y}>v_{\text {th }}$.

We resume only the investigation of the cumulative filamentation/Weibel instability of two symmetric counterstreaming plasmas with positive temperature anisotropies, $v_{\text {th }, y}>v_{\text {th }}$. In the opposite case of $v_{\text {th }, y}<v_{\text {th }}$ and where the anisotropy is less effective and the FI propagating perpendicular to the streaming direction is reduced and even suppressed (Lazar et al. 2006; Stockem \& Lazar 2008), only the Weibel emission along the streaming direc- tion has relevance. This is the Weibel-like mode discussed above.

\subsection{Comparison of the Relaxation Mechanisms}

Let us now compare the maximum growth rates of these different instabilities for the same conditions imposed on the temperature anisotropy and the streaming velocity.

Assuming first that the plasma is hotter along the streaming direction so that $v_{\mathrm{th}, y}>v_{\text {th }}$ and the temperature anisotropy is positive, i.e., $A=\left(v_{\mathrm{th}, y} / v_{\mathrm{th}}\right)^{2}-1>0$, only the FI competes with the TSI and their growth rates are compared in Figure 4, with dashed and solid lines, respectively. The maximum growth rate of the TSI can be larger than that of the FI, but only for a very small anisotropy, $A \rightarrow 0$. In this case, the TSI will be the primary relaxation mechanism for the initial counterstreaming plasma configuration, and this scenario is quite similar to that presented by Schlickeiser \& Shukla (2003) for a beam-plasma system. Due to the electrostatic instability the beam-plasma distribution quickly relaxes to a plateaued distribution in the initial direction of the beam, and after some nonresonant energy exchanges between the background plasma and the plateaued distribution, the plasma distribution can be characterized as anisotropic bi-Maxwellian with two 
characteristic thermal velocities, one is given by the bulk velocity of the beam, and the other one perpendicular to the beam direction is given by the temperature of the background plasma. Finally, this temperature anisotropy is capable to generate Weibel magnetic fields which are evaluated in Schlickeiser \& Shukla (2003). When the temperature anisotropy is negligible, the FI could become faster than the TSI only for very large, fully relativistic streaming velocities, and therefore, the FI will certainly play an important role in the more violent astrophysical phenomena like jets and shock waves in Gamma-Ray Burst sources, etc.

We now consider the effects of a large temperature anisotropy, and remember first that thermal spread along the streaming direction will reduce or evenly suppress the electrostatic instability (TSI), which, otherwise, is very competitive. The numerical solutions displayed in Figure 4, are obtained for temperatures comparable to the average temperature of the IGP, and if we consider large temperature anisotropies, the FI becomes faster (see Figure 4(a)) or even much faster (Figure 4(b)) than the TSI. With the thermal parameters chosen in Figure 4(b), there is no electrostatic instability, the two-stream modes are completely stabilized. But even so, if we consider both sides, (a) and (b), of Figure 4, we should observe that the growth rates of FI are significantly larger than those obtained for the counterstreaming plasmas with isotropic distributions $(A=0)$. This is explained by an enhancing effect due to the temperature anisotropy, which gives rise to the Weibel instability along the same direction, $x$-axis in Figure 1, with the FI. We deal in this case with a cumulative Weibel/FI (Lazar et al. 2006; Stockem \& Lazar 2008) that must be more effective in the process of magnetization and relaxation of a plasma system. Therefore, now we should be more explicit and specify that in Figure 4, the growth rates of this cumulative Weibel/FI are shown (with dashed lines).

On the other hand, if the anisotropy is negative, $A=$ $\left(v_{\text {th }, y} / v_{\text {th }}\right)^{2}-1<0$, the transverse temperature is higher, so that $v_{\text {th }}>v_{\text {th }, y}$, and the WI propagating along the streaming direction can develop faster than the TSI, but only for small streaming velocities and/or for sufficiently large transverse temperatures. This is the case in Figure 3(b), where the growth rates of the WI shown with dotted lines are markedly larger than those of the TSI (solid line). Otherwise, the TSI is fast enough to develop, see in Figure 3(a), relaxing the counterstreaming plasma and heating resonantly the plasma particles to a thermal velocity along the $y$-axis comparable to $v_{0}$ (Schlickeiser \& Shukla 2003). The anisotropy is therefore suppressed and any WI has no chance to arise efficiently. The streaming velocity was assumed initially to be comparable to the average thermal velocity of the IGP $\left(\sim 10^{7} \mathrm{~m} \mathrm{~s}^{-1}\right)$.

Even when they are both propagating along the same direction, these two instabilities are not necessarily in competition, because they perturb the plasma on different (perpendicular) directions, and, in fact, they have different origins. The temperature anisotropy is the origin of WI, while the TSI is generated by the relative motion of counterstreaming plasmas. This case seems to describe only a particular situation because it is conditioned by a negative temperature anisotropy, $v_{\mathrm{th}}>v_{\mathrm{th}, y}$, of the plasma particles. But we should remember that such a condition can be naturally provided as long as the transverse temperature of the plasma streams is increased by the self-generated magnetic field.

The interplay of these instabilities is summarized in Table 1. For slow nonrelativistic plasma flows corresponding to $v_{0}=$ nonrelativistic in Table 1 , and which are relevant for the cosmological scenarios, we have shown the dominance of the magnetic instabilities (FI and WI) against the electrostatic TSI in two specific situations, $\mathrm{WI}_{1}>$ TSI and FI/WI $2>$ TSI. For relativistic streams $\left(v_{0}=\right.$ relativistic $)$, we have only inserted some estimates from the previous works but these cases deserve further and more detailed investigation elsewhere.

\section{SATURATED MAGNETIC FIELD}

We now have a complete overview on how these plasma instabilities are able to relax the anisotropic structures of cosmological plasmas. At least one of the Weibel or filamentation instabilities can be fast enough and more efficient than the electrostatic TSI in the process of relaxation. We must state that each of these two instabilities of Weibel type could become the primary mechanism of the relaxation only in the presence of internal temperature anisotropies, while the TSI is inhibited by thermal effects. Otherwise, for a low temperature of the counterstreaming plasma, the TSI will be markedly faster thermalizing the counterstreaming plasma system, which relaxes to a thermally anisotropic structure and finally, it is the Weibel instability that arises as a secondary mechanism and releases the temperature anisotropy. In this case, the maximum magnetic field reached at the saturation of the Weibel instability was calculated in Schlickeiser \& Shukla (2003), and it is in good agreement with the observations.

In this section, we calculate the magnitudes of the saturated magnetic field obtained for the cases where at least one of the Weibel or filamentation instabilities is faster than the TSI. Then, we compare the results to the observations of cosmological plasma emissions.

The magnetic perturbation grows to large amplitudes and saturates due to the magnetic trapping of plasma particles. The saturation occurs when the magnetic bounce frequency, $\omega_{B}$, increases to a value comparable to the linear growth rate prior to the saturation (Davidson et al. 1972; Califano et al. 1998),

$$
\Gamma_{\max } \simeq \Omega_{e, \max } \equiv\left(\frac{e k_{\max }}{m} \frac{V B_{\max }}{c}\right)^{1 / 2},
$$

where $V$ is the largest characteristic particle velocity perpendicular to the direction of emission of the magnetic instability (Weibel or filamentation). Moreover, as the magnetic field amplitude grows, the transverse deflection of particles becomes stronger (Medvedev \& Loeb 1999) and they are magnetically trapped. In this case, the particle's gyro-radius in the excited magnetic field, namely $\rho=V / \Omega_{e, \max }=e B_{\max } /(m c)$, becomes comparable to the characteristic length scale of the unstable modes, $k_{\max }^{-1}$, yielding (Medvedev \& Loeb 1999; Schlickeiser \& Shukla 2003)

$$
B_{\max } \simeq \frac{m V c k_{\max }}{e} .
$$

Combining now Equations (13) and (14) we find the maximum value of the excited magnetic field,

$$
B_{\max }=\frac{m c}{e} \Gamma_{\max } .
$$

The maximum values of the growth rates, $\Gamma_{\max }$, can be evaluated exactly only numerically, see, for example, the formalism from Lazar (2008). In the following section, we use the appropriate asymptotic approximations of the plasma dispersion function to simplify the dispersion relations (5) and (11), and to derive analytical expressions of the aperiodic solutions and their maxima. 
Table 1

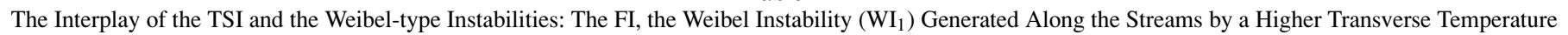
$\left(v_{\mathrm{th}, y}<v_{\mathrm{th}}\right)$, and the Cumulative Filamentation/Weibel Instability $\left(\mathrm{FI} / \mathrm{WI}_{2}\right)$ Generated Perpendicular to the Streams for $v_{\text {th, } y}>v_{\mathrm{th}}$

\begin{tabular}{|c|c|c|c|c|}
\hline \multirow{2}{*}{$\begin{array}{l}\text { Counterstreaming Plasmas } \\
\text { With Thermal Anisotropies }\end{array}$} & \multicolumn{2}{|c|}{$-1<A<0$} & \multicolumn{2}{|c|}{$A>0$} \\
\hline & $v_{\text {th, } y}<v_{\text {th }}$ & $v_{\mathrm{th}, y} \lesssim v_{\mathrm{th}}$ & $v_{\text {th, } y} \gtrsim v_{\text {th }}$ & $v_{\text {th, } y}>v_{\text {th }}$ \\
\hline$v_{0}=$ nonrelativistic & $\begin{array}{c}\mathrm{WI}_{1}>\mathrm{TSI} \\
\mathrm{FI}<\mathrm{TSI}\end{array}$ & $\begin{array}{c}\mathrm{TSI}>\mathrm{FI} \\
\mathrm{TSI}>\mathrm{WI}_{1}\end{array}$ & $\mathrm{TSI}>\mathrm{FI}$ & $\mathrm{FI} / \mathrm{WI}_{2}>\mathrm{TSI}$ \\
\hline$v_{0}=$ relativistic & $\begin{array}{c}\mathrm{WI}_{1}>\mathrm{TSI} \\
(?)\end{array}$ & $\begin{array}{c}\mathrm{FI}>\mathrm{TSI} \\
(?)\end{array}$ & $\begin{array}{c}\mathrm{FI}>\mathrm{TSI} \\
(?)\end{array}$ & $\begin{array}{c}\mathrm{FI} / \mathrm{WI}_{2}>\mathrm{TSI} \\
(?)\end{array}$ \\
\hline
\end{tabular}

\subsection{Original Weibel Instability $\left(v_{0=0}\right)$}

When the streaming velocities are small enough to be neglected, $v_{0} \rightarrow 0$, and the transverse temperature is sufficiently large, $v_{\text {th }}>v_{\text {th }, y}$, so that $\omega>k_{y} v_{\text {th }, y}$, the aperiodic solution of Equation (5) will simplify to

$$
\Gamma \simeq \frac{k_{y} v_{\text {th }}}{\sqrt{2}}\left(1+\frac{k_{y}^{2} c^{2}}{\omega_{p, e}^{2}}\right)^{-1 / 2} .
$$

This coincides to that obtained by Weibel (1959) for a simple thermally anisotropic plasmas with a bi-Maxwellian distribution function. The maximum growth rate is found for $k_{y} \rightarrow \infty$ in Equation (16),

$$
\Gamma_{\max } \simeq \frac{v_{\text {th }} \omega_{p, e}}{\sqrt{2} c} .
$$

By inserting Equation (17) into Equation (15) we obtain the saturated magnetic field

$$
B_{\max }^{0}=\frac{m v_{\mathrm{th}} \omega_{p, e}}{\sqrt{2} e}=\sqrt{4 \pi n}\left(\frac{m v_{\mathrm{th}}^{2}}{2}\right)^{1 / 2}
$$

in terms of the plasma density, $n$, and the transverse kinetic energy of the electrons $E=W / n=m v_{\text {th }}^{2} / 2$. Expression (18) describes the maximum of efficiency in the conversion of the particle kinetic energy into magnetic field energy, $\eta_{B}=$ $B^{2} /\left(4 \pi W_{c}\right)=1$, i.e., the equipartition values of $B$. But this is only an ideal case and we will show in the next that even theoretically, for the Weibel-like mode $\left(v_{0} \neq 0\right)$ this efficiency is lower. Moreover, in numerical simulations of the FI (see, e.g., Califano et al. 1998), which is similar to the Weibel instability, the saturation occurs at subequipartition values of $B, \eta_{B} \simeq$ $0.01-0.1$.

\subsection{Weibel Instability in Counterstreaming Plasmas:

$$
v_{t h}>v_{t h, y}
$$

Due to the internal temperature anisotropies, $v_{\text {th }}>v_{\text {th }, y}$, the Weibel unstable modes arise independently of the TSI. For plasmas with very large anisotropies, $v_{\text {th }} \gg v_{\text {th }, y}$, we use in Equation (5) the asymptotic approximation of plasma dispersion function for large arguments $f_{1,2} \gg 1$, and find the aperiodic solutions

$$
\operatorname{Im} \omega=\Gamma \simeq k_{y}\left[\frac{v_{\mathrm{th}}^{2}}{2\left(1+k_{y}^{2} c^{2} / \omega_{p, e}^{2}\right)}-v_{0}^{2}\right]^{1 / 2},
$$

as long as the wavenumber is less than a cutoff value

$$
k_{y} \leqslant k_{c, 1} \simeq \frac{\omega_{p, e}}{c}\left(\frac{v_{\mathrm{th}}^{2}}{2 v_{0}^{2}}-1\right)^{1 / 2},
$$

and

$$
v_{\text {th }} \geqslant \sqrt{2} v_{0} .
$$

For wavenumbers greater than $k_{c, 1}$, the modes are stabilized by the resonant Landau damping with the rate given by the same relation (19). Otherwise, for low wavenumbers $0<k \leqslant k_{c, 1}$, the Weibel modes reaches a maximum growth rate

$$
\Gamma_{\max }=\Gamma\left(k_{y, \max }\right)=\frac{\omega_{p, e}}{c}\left(\frac{v_{\mathrm{th}}}{\sqrt{2}}-v_{0}\right),
$$

for

$$
k_{y, \max }=\frac{\omega_{p, e}}{c}\left(\frac{v_{\mathrm{th}}}{\sqrt{2} v_{0}}-1\right)^{1 / 2}<k_{c, 1} .
$$

But, if we look carefully to the assumption of large arguments of the plasma dispersion function, $\Gamma / k_{y} v_{\text {th }} \geqslant 1$, from above, we find that the wavenumber must be less than

$$
k_{y} \leqslant k_{c, 2}=\frac{\omega_{p, e}}{c}\left\{\frac{v_{\mathrm{th}}^{2}}{2\left[\left(v_{\mathrm{th}, y}+v_{0}\right)^{2}+v_{0}^{2}\right]}-1\right\}^{1 / 2}<k_{c, 1},
$$

as long as

$$
v_{\text {th }} \geqslant \sqrt{2}\left[\left(v_{\text {th }, y}+v_{0}\right)^{2}+v_{0}^{2}\right]^{1 / 2} .
$$

This is the new minimum limit of the transverse thermal velocity, which is greater than $\sqrt{2} v_{0}$ and much greater than $v_{\text {th }, y}$, so that both conditions $v_{\text {th }}>v_{\text {th, } y}$ and (21) are fulfilled at the same time. But the new maximum limit for the wavenumber, given by Equation (24), will limit the Weibel growth rate to

$$
\Gamma_{\max }=\Gamma\left(k_{c, 2}\right)=k_{c, 2}\left(v_{\mathrm{th}, y}+v_{0}\right) .
$$

With Equation (26) substituted into Equation (15), the saturated magnetic field reads

$$
B_{\max }=B_{\max }^{0}\left(v_{\mathrm{th}, y}+v_{0}\right)\left[\frac{1}{\left(v_{\mathrm{th}, y}+v_{0}\right)^{2}+v_{0}^{2}}-\frac{2}{v_{\mathrm{th}}^{2}}\right]^{1 / 2} .
$$

In order to see how important the "correction" found in Equation (27) with respect to Equation (18) is, we consider a particular situation but close to our case here, when the plasma has a very large internal anisotropy so that it is (much) hotter transversally to the streaming direction, $v_{\text {th }, y} \sim v_{0}<v_{\text {th }}$. With this assumption and taking condition (25) with $v_{0} / v_{\text {th }} \equiv$ $\mu<\sqrt{10} / 10$, the magnetic field saturation occurs at slightly subequipartition values

$$
\begin{aligned}
\sqrt{\eta} & =B_{\max } / B_{\max }^{0} \\
& =\frac{2}{\sqrt{5}}\left(1-10 \mu^{2}\right)^{1 / 2} \simeq 0.89-4.47 \mu^{2}<0.89 .
\end{aligned}
$$




\subsection{Cumulative Weibel/FI}

When the plasma is hotter along the streaming direction $\left(v_{\text {th }, y}>v_{\text {th }}\right)$, the FI is enhanced by the Weibel effect leading to significantly large growth rates. For sufficiently large temperature anisotropies, $A \gg 1$, we use the asymptotic approximation for large arguments of the plasma dispersion function in Equation (11), and find the analytical expression of the growth rate

$\Gamma \simeq k_{x} v_{\text {th }}\left[\frac{A+2\left(v_{0} / v_{\mathrm{th}}\right)^{2}}{1+\left(k_{x} c / \omega_{p, e}\right)^{2}}\right]^{1 / 2} \simeq \frac{\omega_{p, e}}{c}\left[\frac{v_{\mathrm{th}}^{2}+2 v_{0}^{2}}{1+\left(\omega_{p, e} / k_{x} c\right)^{2}}\right]^{1 / 2}$.

The maximum is reached for very large wavenumbers

$$
\Gamma_{\max } \simeq \frac{\omega_{p, e}}{c}\left(v_{\mathrm{th}}^{2}+2 v_{0}^{2}\right)^{1 / 2},
$$

and the saturated magnetic field

$$
B_{\max }=\sqrt{8 \pi n}\left[\frac{m\left(v_{\mathrm{th}, y}^{2}+2 v_{0}^{2}\right)}{2}\right]^{1 / 2} .
$$

Otherwise, if the cumulative Weibel/FI is still not so fast, then the TSI develops first relaxing the counterstreams to a thermally anisotropic distribution, which is unstable against the excitation of the simple Weibel instability. In this case, the magnetic field at saturation was calculated by Schlickeiser \& Shukla (2003).

\subsection{Comparison with the Intergalactic Magnetic Field}

Medium-density ( $n \sim 10^{-4} \mathrm{~cm}^{-3}$ ) and relatively hot plasmas may be found in intergalactic space, and the high-resolution observations of the synchrotron intergalactic sources have proved the existence of a diffuse universal intergalactic magnetic field (Donahue et al. 2003). Whatever the origin of the cosmological magnetic field is (either it is primordial or it was amplified to its present strength by diverse mechanisms driven by cosmic plasma turbulence), we are presently capable to detect and measure these fields even for extremely distant radio-galaxies of a large red-shift, $z>5$ (van Breugel et al. 1999). There are micro-Gauss-level fields which are common in spiral galaxy disks and halos, and which appear to be a common property of the extragalactic or intra-cluster medium as well (Kronberg 1994; Widrow 2002). Moreover, recent advances in observational methods have revealed large-scale structures with reversals of the direction of magnetic fields (Han et al. 2006) quite similar to those created by the instabilities of the Weibel type. Indeed, it was already shown by Schlickeiser (2005) that the Weibel mechanism is capable of generating magnetic fields from the plasma Debye scale up to cosmological scales.

Here, we estimate the saturated magnetic field that scales by the maximum growth rate of the instability, as given in Equation (15), and compare to the values known as representative for the cosmic space. The maximum growth rate value reached by the instability at the saturation, is provided exactly (numerically) in the Figures 3 and 4 for each case apart, or it can be determined approximatively using the analytical formalism from above.

If we give initial conditions to the Weibel instability to arise along the streaming direction, so that, $v_{\mathrm{th}, y} \sim v_{0} \simeq 10^{7} \mathrm{~m} \mathrm{~s}^{-1}$, and the thermal anisotropy is moderately large, $v_{\mathrm{th}} / v_{\mathrm{th}, y}=5$, this corresponds to the lowest growth rate of the WI in Figure 3, but still higher than the TSI growth rate. Using Equation (27), the saturated magnetic field is found as $B_{\max } \simeq 3 \times 10^{-6} \mathrm{G}$. Even when the saturation occurs at slightly subequipartition values of
$\mathrm{B}, \eta_{B} \simeq 0.01-0.1$, the saturated magnetic fields generated by the Weibel instability in this case, will reach values not less than $10^{-7} \mathrm{G}$.

For the opposite case, when an internal temperature anisotropy is present but it is favorable to the formation of a cumulative filamentation/Weibel instability, we consider two representative situations corresponding to those presented in Figure 4(a) and (b). If we consider Figure 4(a), and take, for example, $v_{\text {th }, y} \sim v_{0} \simeq 10^{7} \mathrm{~m} \mathrm{~s}^{-1}$, and the anisotropy $v_{\text {th }, y} / v_{\text {th }}=5$, the saturated magnetic field obtained from Equation (31) is $B_{\max } \simeq 2 \times 10^{-6} \mathrm{G}$. For the second case, in Figure 4(b), we take $v_{\text {th }} \sim v_{0} \simeq 10^{7} \mathrm{~m} \mathrm{~s}^{-1}$, the anisotropy $v_{\text {th }, y} / v_{\text {th }}=5$, and from Equation (31) we find $B_{\max } \simeq 6 \times 10^{-6}$ G. Both of these two values corresponding to the cumulative filamentation/ Weibel instability are comparable to the first value obtained for the WI, because their growth rates are comparable. However, in the last case, when the filamentation and Weibel effects cumulate, the saturated magnetic field is several times larger, and even for a conversion efficiency $\eta_{B} \simeq 0.01-0.1$ it can easily reach micro-Gauss levels, $B=\eta_{B}^{1 / 2} B_{\max } \simeq(0.6-1.9) \times 10^{-6} \mathrm{G}$.

\section{DISCUSSIONS AND CONCLUSIONS}

The idea that the WI, provoked by a plasma temperature anisotropy, could explain the origin of the cosmological magnetic field has been launched relatively recently. There were several scenarios, either the instability is generated as a secondary mechanism of relaxation (after the TSI) of the high Mach number flows in intergalactic medium (Schlickeiser \& Shukla 2003), or in the steep temperature gradients associated with the cold fronts discovered in cluster of galaxies (Okabe \& Hattori 2003), or it is expected to develop at shock waves associated with the formation of galaxies or cluster of galaxies (Fujita \& Kato 2006).

In the present paper, we have extended the investigations to any form of the Weibel-type instability, including WI and FI, and which are capable to develop in more complex plasma systems. Therefore, we have chosen to investigate a more complex structure of a counterstreaming plasma with intrinsic temperature anisotropies, and with physical parameters derived from the observations of the IGP. Two types of plasma instabilities have been identified as possible mechanisms for releasing the excess of free energy stored in such anisotropic plasma. The first are the electromagnetic instabilities of Weibel type, which are represented by either the Weibel modes generated by an excess of transverse temperature with respect to the streaming direction, or by the cumulative filamentation/Weibel modes when the plasma is hotter along the streaming direction. They compete with the two-stream electrostatic instability, which seems to be very fast under the presumptive conditions fixed for the plasma system (Schlickeiser \& Shukla 2003).

We have preserved some of the parameters and conditions used by Schlickeiser \& Shukla (2003), but here we have shown for the first time, that, as long we admit the existence of internal temperature anisotropies of the interpenetrating plasmas, the instabilities of Weibel type could be easily faster than the principal competitor, which is the TSI, and thus become the primary relaxation mechanism of the plasma anisotropy. For each of these cases, we have calculated the growth rates and the maximum magnetic field produced at the saturation. These magnetic field values are consistent with the magnetic field values, in the range $10^{-7}-10^{-5} \mathrm{G}$, derived from the Faraday rotation measure of the linearly polarized emission of galactic 
or extragalactic sources (Widrow 2002; Wielebinski \& Beck 2005). Thus, the robustness and the occurrence of the magnetic instabilities of the Weibel type for the generation of the seed magnetic field in the early Universe is guaranteed.

The authors acknowledge financial support from the Research Foundation Flanders - FWO Belgium, the Deutsche Forschungsgemeinschaft through the Sonderforschungsbereich 591, Schl 201/17-1 and Schl 201/21-1. These results were obtained in the framework of the projects GOA/2009009 (K. U. Leuven), G.0304.07 (FWO-Vlaanderen), and C 90205 (ESA Prodex 9). Financial support by the European Commission through the SOLAIRE Network (MTRNCT-2006-035484) is gratefully acknowledged. The numerical results were obtained on the HPC cluster VIC of the K. U. Leuven.

We are grateful to the anonymous referee for his constructive comments and suggestions.

\section{REFERENCES}

Battefeld, D., Battefeld, T., Wesley, D. H., \& Wyman, M. 2008, J. Cosmol. Astropart. Phys. JCAP02(2008)001

Bergman, J., \& Eliasson, B. 2001, Phys. Plasmas, 8, 1482

Bret, A., \& Deutsch, C. 2006, Phys. Plasmas, 13, 022110

Bret, A., Firpo, M.-C., \& Deutsch, C. 2004, Phys. Rev. E, 70, 046401

Bret, A., Firpo, M.-C., \& Deutsch, C. 2005, Phys. Rev. Lett., 94, 115002

Brügen, M., Ruszkowski, M., Simionescu, A., Hoeft, M., \& Dalla Vecchia, C. 2005, ApJ, 631, L21

Califano, F., Pegoraro, F., Bulanov, S. V., \& Mangeney, A. 1998, Phys. Rev. E, 57,7048

Davidson, R. C., Hammer, D. A., Haber, I., \& Wagner, C. E. 1972, Phys. Fluids, 15,317
Donahue, M., Daly, R. A., \& Horner, D. J. 2003, ApJ, 584, 543

Fiore, M., Silva, L. O., Ren, C., Tzoufras, M. A., \& Mori, W. B. 2006, MNRAS, 372, 1851

Fried, B. D. 1959, Phys. Fluids, 2, 337

Fried, B. D., \& Conte, S. D. 1961, The Plasma Dispersion Function (New York: Academic Press)

Fujita, Y., \& Kato, T. N. 2006, Astron. Nachr., 327, 443

Han, J. L., Manchester, R. N., Lyne, A. G., Qiao, G. J., \& van Straten, W. 2006, ApJ, 642, 868

Harrison, E. R. 1970, Mon. Not. R. Astron. Soc., 147, 279

Kronberg, P. P. 1994, Rep. Prog. Phys., 57, 325

Lazar, M. 2008, Phys. Lett. A, 372, 2446

Lazar, M., Schlickeiser, R., \& Shukla, P. K. 2006, Phys. Plasmas, 13, 102107

Medvedev, M. V., \& Loeb, A. 1999, ApJ, 526, 697

Medvedev, M. V., Silva, L. O., \& Kamionkowski, M. 2006, ApJ, 642, L1

Melrose, D. B. 1989, in Instabilities in Space and Laboratory Plasmas (New York: Cambridge Univ. Press)

Miniati, F. 2002, MNRAS, 337, 199

Okabe, N., \& Hattori, M. 2003, ApJ, 599, L964

Okada, T., Yabe, T., \& Niu, K. 1977, J. Phys. Soc. Jpn., 43, 1042

Parker, E. N. 1955, ApJ, 122, 293

Rees, M. J. 1987, Q. J. R. Astron. Soc., 28, 197

Sakai, J., Schlickeiser, R., \& Shukla, P. K. 2004, Phys. Lett. A, 330, 384

Schaefer-Rolffs, U, \& Schlickeiser, R. 2005, Phys. Plasmas, 12, 022104

Schlickeiser, R. 2004, Phys. Plasmas, 11, 5532

Schlickeiser, R. 2005, Plasma Phys. Control. Fusion, 47, 205

Schlickeiser, R., \& Shukla, P. K. 2003, ApJ, 599, L57

Schlickeiser, R., Sievers, A., \& Thiemann, H. 1987, A\&A, 182, 21

Steenbeck, M., \& Krause, F. 1968, Astron. Nachr., 291, 49

Stockem, A., \& Lazar, M. 2008, Phys. Plasmas, 15, 014501

Taylor, G. B., Barton, E. J., \& Ge, J.-P. 1994, AJ, 107, 1942

Vainshtein, S. I., \& Ruzmaiken, A. A. 1971, Soviet Ast., 15, 714

van Breugel, W., De Breuck, C., Stanford, S. A., Stern, D., Röttgering, H., \& Miley, G. 1999, ApJ, 518, L61

Weibel, E. S. 1959, Phys. Rev. Lett., 2, 83

Widrow, L. M. 2002, Rev. Mod. Phys., 74, 775

Wielebinski, R., \& Beck, R. (ed.), 2005. Cosmic Magnetic Fields (Heidelberg: Springer) 